\title{
The Canadian Preterm Birth Network: a study protocol for improving outcomes for preterm infants and their families
}

\author{
Prakesh S. Shah MD MSc, Sarah D. McDonald MD MSc, Jon Barrett MD, Anne Synnes MDCM MHSc, \\ Kate Robson MEd, Jonathan Foster CPA CMA, Jean-Charles Pasquier MD PhD, K.S. Joseph MD PhD, \\ Bruno Piedboeuf MD, Thierry Lacaze-Masmonteil MD PhD, Karel O'Brien MD, \\ Sandesh Shivananda MD MSc, Nils Chaillet PhD, Petros Pechlivanoglou PhD; for the Canadian Preterm \\ Birth Network Investigators*
}

\section{Abstract}

Background: Preterm birth (birth before 37 wk of gestation) occurs in about $8 \%$ of pregnancies in Canada and is associated with high mortality and morbidity rates that substantially affect infants, their families and the health care system. Our overall goal is to create a transdisciplinary platform, the Canadian Preterm Birth Network (CPTBN), where investigators, stakeholders and families will work together to improve childhood outcomes of preterm neonates.

\begin{abstract}
Methods: Our national cohort will include 24 maternal-fetal/obstetrical units, 31 neonatal intensive care units and 26 neonatal follow-up programs across Canada with planned linkages to provincial health information systems. Three broad clusters of projects will be undertaken. Cluster 1 will focus on quality-improvement efforts that use the Evidence-based Practice for Improving Quality method to evaluate information from the CPTBN database and review the current literature, then identify potentially better health care practices and implement identified strategies. Cluster 2 will assess the impact of current practices and practice changes in maternal, perinatal and neonatal care on maternal, neonatal and neurodevelopmental outcomes. Cluster 3 will evaluate the effect of preterm birth on babies, their families and the health care system by integrating CPTBN data, parent feedback, and national and provincial database information in order to identify areas where more parental support is needed, and also generate robust estimates of resource use, cost and cost-effectiveness around preterm neonatal care.
\end{abstract}

Interpretation: These collaborative efforts will create a flexible, transdisciplinary, evaluable and informative research and qualityimprovement platform that supports programs, projects and partnerships focused on improving outcomes of preterm neonates.

$\mathrm{P}$ reterm birth, defined as birth occurring before 37 weeks' gestation, occurs in about $8 \%$ of pregnancies in Canada ${ }^{1}$ and has a life-long impact on babies, their families and society. As the leading cause of infant death, cerebral palsy and disability, preterm birth is estimated to cost the Canadian health care system over $\$ 8$ billion per year. ${ }^{2}$ Previous studies by our group showed that, by applying collaborative, integrated quality improvement via networking, the incidence of major neonatal morbidity decreased by $20 \%-50 \%$ in infants less than 29 weeks of gestational age. ${ }^{3-5}$ Outcome improvements to date have used data platforms limited to neonatal and neonatal follow-up research ${ }^{6,7}$ despite mounting evidence that events before and during pregnancy can have life-long implications for the child. ${ }^{7,8}$ Furthermore, our platforms were missing data regarding family integration into preterm birth care, which can positively affect neonatal outcomes. $^{9}$
The Canadian Preterm Birth Network (CPTBN), described in this protocol, is funded by the Canadian Institutes of Health Research and aims to expand our existing neonatal platforms to develop a pan-Canadian network consisting of a team with expertise in maternal-fetal medicine, obstetrics, neonatology,

\section{Competing interests: None declared.}

${ }^{*}$ A complete listing of Canadian Preterm Birth Network Investigators is provided in Appendix 1 (available at www.cmajopen.ca/content/6/1/E44/ suppl/DC1).

This article has been peer reviewed.

Correspondence to: Prakesh Shah, prakeshkumar.shah@ sinaihealthsystem.ca

CMAJ Open 2018. DOI:10.9778/cmajo.20170128 
pediatrics, neonatal follow-up, epidemiology, health economics and health informatics as well as parents, nurses, other allied health care professionals, national/provincial organizations and policy-makers. The primary goal of the CPTBN is to translate knowledge generated by clinical, quality-improvement and health care services research into optimal preterm birth care practices and policies that substantially improve both short- and long-term outcomes. In this protocol, we describe the use of the CPTBN to implement and assess quality improvement; to evaluate the effect of maternal, neonatal and developmental interventions; and to generate estimates of the economic impact of preterm birth. We also provide the reader with the framework for a flagship national research program designed to address gaps in perinatal-neonatal research and improve Canadian neonatal and family outcomes over the next 5 years.

\section{Methods}

\section{Study design}

The CPTBN team will collect maternal and infant data for Canadian preterm infants and will use the data to perform research projects in 3 integrated research clusters: 1) evidence-based quality improvement, 2) association of maternal, perinatal and neonatal care with outcomes and 3) economics, resource use and surveillance of preterm birth. The CPTBN research program is designed to be flexible and to allow for new projects, project modification, collaborations and partnerships. The 3 research clusters will function as a platform for ongoing integrated comparative effectiveness research, randomized controlled trials, translational studies, proof-ofconcept studies, health services evaluation and programmatic evaluations. The CPTBN will be governed by a steering committee, a director and 4 advisory boards, as outlined in Appendix 2 (available at www.cmajopen.ca/content/6/1/E44/ suppl/DC1).

\section{Setting}

The CPTBN will expand the efforts of the Canadian Neonatal Network (CNN) and affiliated neonatal networks from 2017 to 2021, with specific research projects spread over the study duration as outlined in Figure 1. The CNN collects and maintains data from all 31 level 3 neonatal intensive care units in Canada and is used for benchmarking, collaborative research, quality improvement, training and advocacy. . $^{3,9-11}$ Furthermore, robust linkages exist between the $\mathrm{CNN}$ and its associated databases: the Canadian Neonatal Follow-Up Network (www.cnfun.ca/), which includes 26 neonatal follow-up programs, the Canadian Neonatal Transport Network and the Canadian Pediatric Surgery Network (www.capsnetwork.org/). The CPTBN will incorporate all existing networks and will expand to include the maternal-fetal medicine/obstetrics community from 24 Canadian tertiary perinatal units (Figure 2). In addition, to obtain data on outcomes, use of health care services in early childhood and social determinants of health for all preterm infants, we are developing linkages with the following databases: BORN (Better Outcomes Registry \& Network)

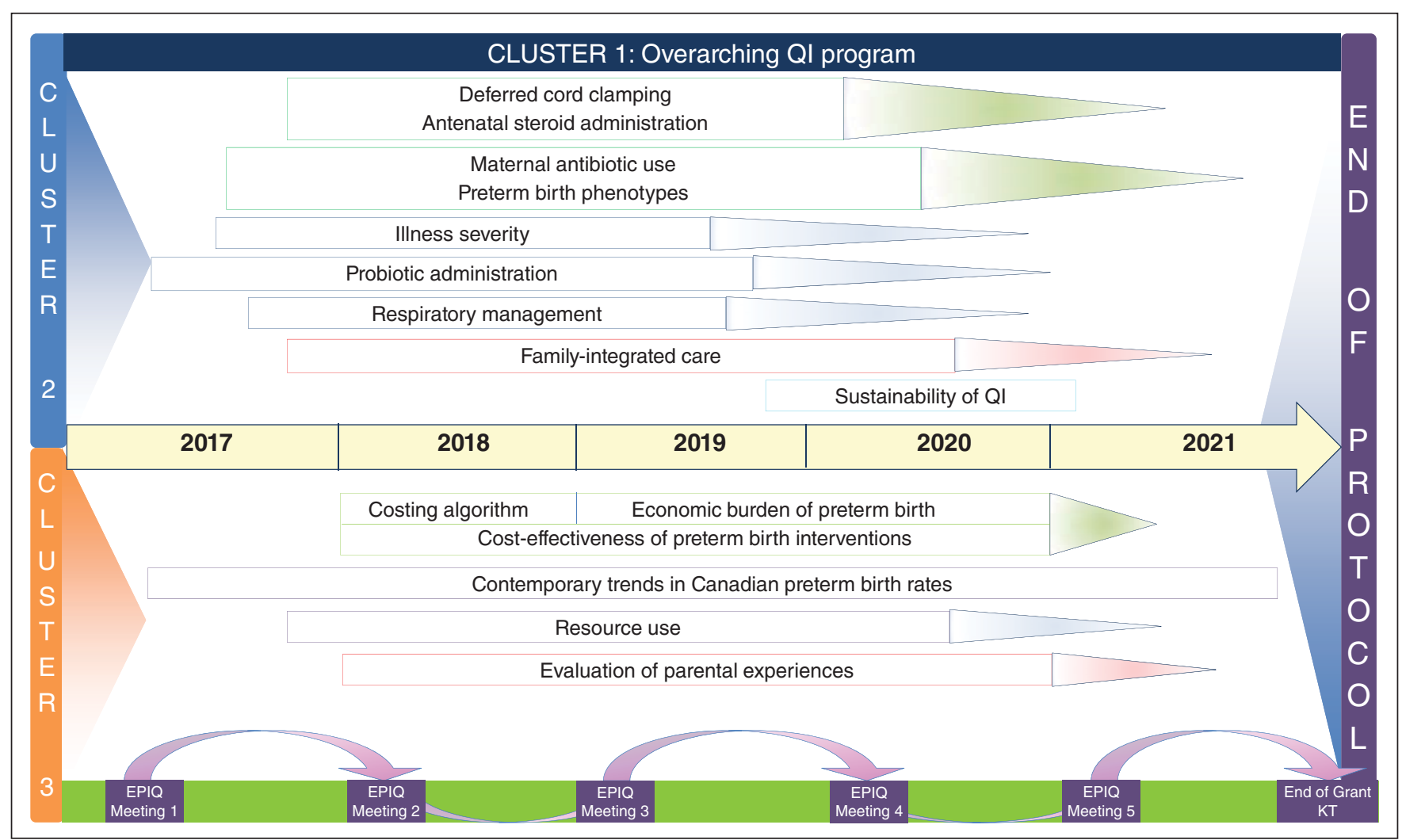

Figure 1: Approximate timeline of Canadian Preterm Birth Network research projects. Note: EPIQ = Evidence-based Practice for Improving Quality, KT = knowledge translation, QI = quality improvement. 
Ontario (https://www.bornontario.ca/), the Nova Scotia Atlee Perinatal Database (http://rcp.nshealth.ca/atlee-database), the Alberta Perinatal Health Program (http://aphp.dapasoft.com/ Lists/HTMLPages/index.aspx), the British Columbia Perinatal Data Registry (www.perinatalservicesbc.ca/), the Quebec Pregnancy Cohort (https://www.bridgetodata.org/node/1013) and the Perinatal Program Newfoundland Labrador (www. easternhealth.ca/Professionals.aspx?d=1\&id=1972\&p=81).

\section{Data collection}

The coordination centre for the CPTBN will be located at the Maternal-Infant Care Research Centre at Mount Sinai Hospital, Toronto. The centralized data management system conforms to the regulations of both the Health Information Protection Act and the Personal Information Protection and Electronic Documents Act and is approved by the Mount Sinai Hospital Research Ethics Board for collecting, developing and hosting the CPTBN data set to ensure data integrity and security.

We will collect data for all preterm births occurring between January 2018 and December 2020. Because of the large number of preterm births in Canada, infants will be strati- fied into 3 gestational age groups: less than 29 weeks (infants who require the most resources and are at the highest risk for adverse outcomes), 29-33 weeks (infants who are moderately preterm and could be managed in level 2 units) and 34-36 weeks (infants who typically do not require intensive care). The source of data for each gestational age group also varies.

\section{Sources of data}

The variable and outcome data collected by the CPTBN are described in detail in the CNN's Abstractor's Manual. ${ }^{12}$ They include mother and baby characteristics, antenatal care, delivery details, neonatal intensive care unit care, follow-up care, maternal outcomes and neonatal outcomes. An internal audit confirmed that the $\mathrm{CNN}$ database is valid and reliable. ${ }^{6}$

\section{Cluster 1: evidence-based quality improvement}

Canadian initiatives using the Evidence-based Practice for Improving Quality (EPIQ) method led to a statistically significant improvement in neonatal outcomes. ${ }^{3-5}$ To build on those improvements, the CPTBN protocol will expand the use of EPIQ methods to maternal-fetal medicine/obstetrics care practices and family-integrated care practices.

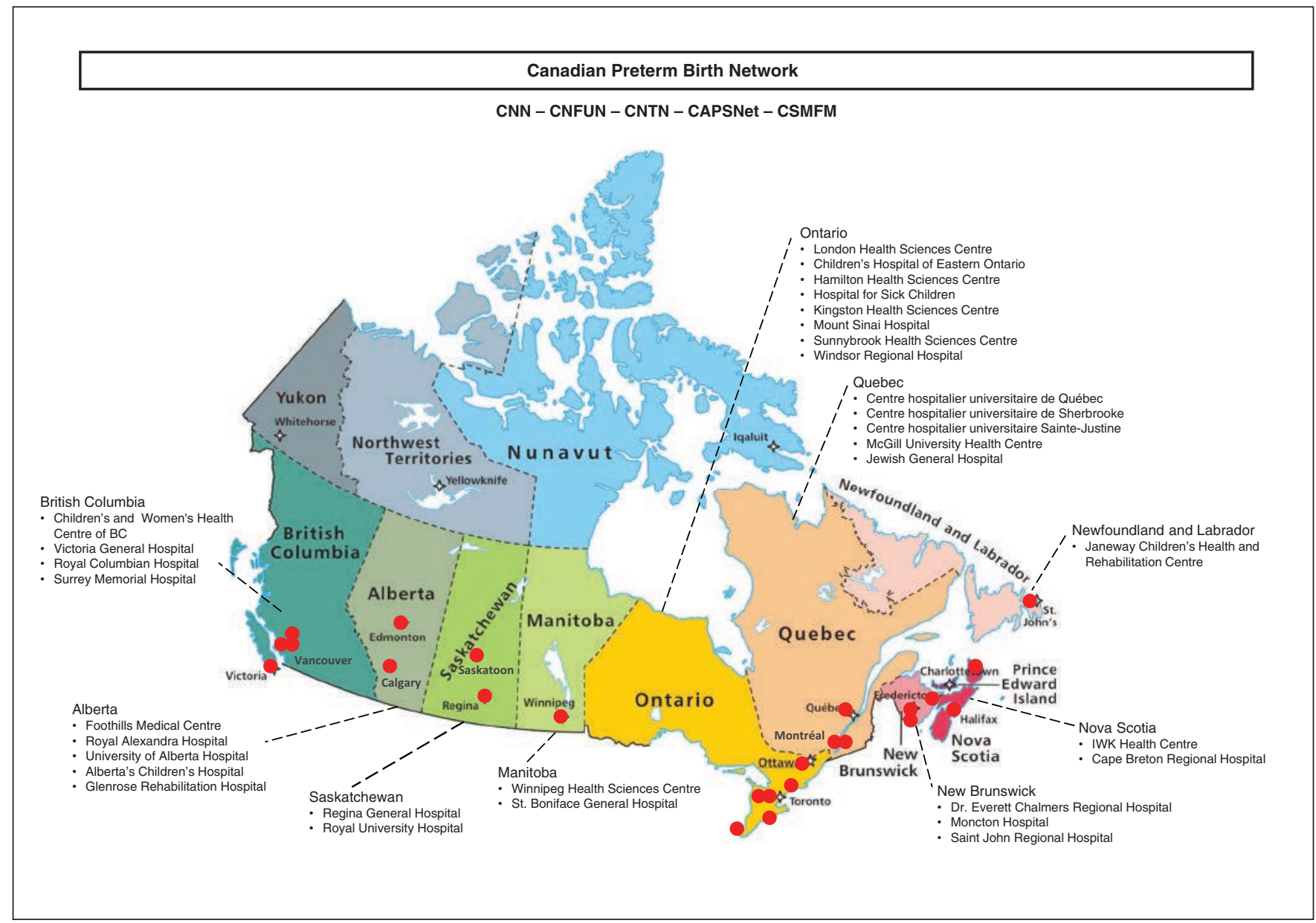

Figure 2: Map of the Canadian Preterm Birth Network. Red dots indicate approximate location of the 31 participating hospitals. Note: CAPSNet = Canadian Pediatric Surgery Network, CNFUN = Canadian Neonatal Follow-Up Network, CNN = Canadian Neonatal Network, CNTN = Canadian Neonatal Transport Network, CSMFM = Canadian Society of Maternal Fetal Medicine. 


\section{Outcomes following quality improvement}

Neonatal outcomes in Canada still lag behind those in other industrialized nations ${ }^{13}$ yet there have been no large-scale quality-improvement efforts for women at risk for giving birth prematurely using local maternal-fetal medicine/obstetrics data. EPIQ methods will be implemented by transdisciplinary investigators at each site who are trained to conduct twiceyearly plan-do-study-act cycles. ${ }^{14,15}$ Investigators will use local data and best available knowledge in perinatal-neonatal care to select and implement quality-improvement activities, then develop and collect process indicator information pertaining to their quality-improvement cycles. Every 6 months, the CPTBN will provide sites with their outcomes, which will be used to compare their performance to the national benchmark.

\section{Institutional factors associated with successful quality improvement}

Simultaneously, we will work to identify facilitators of and barriers to implementation of quality-improvement projects at the institutional level by conducting focus groups, surveys, site visits and discussions with front-line staff and families at units to pinpoint items that may affect implementation of these projects.

\section{Cluster 2: association of maternal, perinatal and neonatal care with outcomes}

Despite a lack of data from large multicentre studies, established and emerging maternal, perinatal and neonatal practices are variably implemented in preterm birth care centres across Canada. In cluster 2, we will identify variable practices and associate the variability to neonatal outcomes.

\section{Deferred cord clamping}

Use of deferred cord clamping is markedly variable in Canada (30\%-40\% among preterm infants). ${ }^{16} \mathrm{We}$ will collect information about the interval between birth and cord clamping, and reasons for immediate cord clamping. We will calculate the rate of change in implementation of deferred cord clamping over the 3-year study period and link data about this practice with neonatal outcomes.

\section{Antenatal steroid administration}

Optimal prenatal administration of steroids to women at high risk for giving birth prematurely can improve survival free of major morbidity in preterm infants. ${ }^{17,18}$ However, there are concerns regarding adverse effects of steroids on the developing brain. ${ }^{19}$ We will determine whether optimal antenatal administration of steroids is associated with improved neurodevelopmental outcomes for neonates.

\section{Maternal antibiotic use}

Mothers share their microbes with their infants, and alteration of the early infant gut microbiome is correlated with the development of childhood obesity, asthma, allergies and type 1 diabetes. ${ }^{8,20,21} \mathrm{We}$ will collect data on the administration of antibiotics to women before delivery and study the association between perinatal use of antibiotics and neonatal and neurodevelopmental outcomes.

\section{Preterm birth phenotypes}

Evidence suggests that preterm birth is a syndrome attributable to multiple pathological processes ${ }^{22,23}$ and that neonatal outcomes may be different based on the reasons for preterm birth. We will collect data on 7 categories of preterm birth (premature rupture of membranes, infection, hemorrhage, hypertensive disorders of pregnancy, other forms of medically indicated preterm birth, multiple births and idiopathic preterm birth) and attempt to link these to neurodevelopmental outcomes.

\section{Illness severity}

The management of threatened preterm labour has improved along with neonatal practices in the first ("golden") hour after birth, ${ }^{24}$ with gentler handling of neonates; however, the impact on illness severity and neurodevelopmental outcome is unknown. The CPTBN will collect data regarding peripartum management strategies (i.e., peripartum interventions, golden hour management) and correlate these with neonatal illness severity on admission to the neonatal intensive care unit (defined by Score for Neonatal Acute Physiology Perinatal Extension ${ }^{25}$ and Transport Risk Index of Physiologic Stability ${ }^{26}$ ) and neonatal and neurodevelopmental outcomes.

\section{Probiotic administration}

Probiotic administration to preterm neonates may reduce rates of necrotizing enterocolitis ${ }^{27}$ and nosocomial infection, ${ }^{28}$ yet only $30 \%$ of Canadian neonates receive probiotics. ${ }^{16} \mathrm{We}$ will attempt to link receipt of probiotics during the neonatal intensive care unit stay as well as type, form (powder v. liquid), duration and timing of probiotic use with neonatal and neurodevelopmental outcomes.

\section{Respiratory management}

The need for and duration of invasive respiratory support after preterm birth is associated with bronchopulmonary dysplasia and other complications. ${ }^{29}$ However, the impact of different types of respiratory support on resource use and neurodevelopmental outcomes is unknown. ${ }^{30-32} \mathrm{We}$ will compare 2 protocols for the provision of respiratory support before intubation and after first extubation in preterm neonates using integrated comparative effectiveness research methods.

\section{Family-integrated care}

Integration of the family in the care of preterm neonates, particularly early parental engagement, skin-to-skin care and parent education, may improve outcomes of preterm neonates. ${ }^{9}$ However, the minimal "dosage" of these interventions is unknown. The CPTBN will evaluate the number of hours of parent involvement, skin-to-skin care and parent education that are associated with subsequent positive neonatal and neurodevelopmental outcomes.

\section{Sustainability of quality improvement}

Sustainability of quality-improvement initiatives is an increasing concern for funders and stakeholders. The CPTBN will use standard methodology to evaluate the sustainability of quality-improvement initiatives in perinatology-neonatology. 


\section{Cluster 3: economics, resource use and surveillance of preterm birth}

Current methods for estimating the cost of preterm birth in Canada exclude maternal data, rely on simulation modelling, use crude provincial resource use data and lack both depth and inclusivity over time and across different subgroups. ${ }^{33}$

\section{Costing algorithm}

We will develop a costing algorithm using a combination of prospective and retrospective data on patient-level resource use and resource-specific unit costs attained by purposive and representative sampling in Canada. We will collect data on resource use from the perinatal to postneonatal period from resources such as the CNN and the Discharge Abstract Database of the Canadian Institute for Health Information. We will calculate unit costs using billing data or attributable costing methods, then generate a comprehensive algorithm compatible with data analysis programs.

\section{Economic burden of preterm birth}

The cost algorithm will allow us to identify the economic burden of the components of preterm birth care, the entirety of preterm birth care and the variation in costs by subpopulations and provinces/territories in Canada. We will use retrospective and prospective nested cohort studies to assess health care costs accrued by infants and parents for 2 years following birth.

\section{Cost-effectiveness of preterm birth interventions}

We will develop a system-wide economic decision model that will simulate hypothetical dyads of mothers/babies and will follow the dyad throughout the ante-, peri- and postnatal continuum. Data on resource use, unit costs and health outcomes from the CPTBN, other Canadian organizations, systematic reviews and expert consultations will be used in our model. From this model, we will identify the interventions that offer the best outcomes and are economically efficient.

\section{Contemporary trends in Canadian preterm birth rates}

Preterm birth surveillance in Canada lacks timely and accurate information on preterm birth rates. The most recent estimate of preterm birth rate $(7.7 \%)$ is from 2010 and excludes Ontario births. ${ }^{1}$ The CPTBN will calculate rates of both preterm birth and medically indicated preterm birth (to identify system issues) in each province/territory using birth cohorts from 2017 to 2021 (supplemented with data from the 20032016 birth cohorts) from the Discharge Abstract Database and the MED-ÉCHO (Maintenance et exploitation des données pour l'étude de la clientèle hospitalière) database in Quebec.

\section{Resource use}

The number of infants born moderately or slightly prematurely (32-36 wk of gestational age) has risen recently, ${ }^{34}$ creating a need to determine medical resource use and predictors of increased resource use for these groups. We will identify the patterns of use of health care resources during the hospital stay and after discharge for preterm infants for 2 years following birth.

\section{Evaluation of parental experiences}

Integration of the family into preterm infant care is important to achieve the best neonatal outcomes, ${ }^{9}$ but feedback regarding parent satisfaction with care is not routinely collected. In addition, the family's postdischarge experience is not regularly communicated to the neonatal intensive care unit or neonatal follow-up programs. We will develop an electronic Webbased platform to capture parental experiences with the medical system from birth to 2 years after discharge from the neonatal intensive care unit.

\section{Statistical analysis}

During the 3 years of data collection, we will prospectively collect information for roughly 1500 infants less than 29 weeks of gestational age per year. Assuming a mortality rate of $15 \%$ and $20 \%$ loss to follow-up, we expect that we will collect neonatal and neurodevelopmental outcome data for 1000 infants per year. We will compare the characteristics of patients lost to follow-up with those of patients who are followed. We will not impute any data that are not available. A sample this size will provide greater than $90 \%$ power to detect a $30 \%$ relative increase in survival free of neonatal morbidity (baseline $47 \%$ $61 \%$ ) and $30 \%$ relative decrease in significant neurodevelopmental impairment (baseline 17\%-11.9\%) in the last year compared to the first year at a significance level of 0.05 after accounting for clustering within each site. ${ }^{35}$ Under the same assumptions, a conservative estimate of $20 \%$ change in the 2 outcomes will have greater than $80 \%$ power. Sample size for nested cohorts will be determined based on feasibility. We plan to use specific quality-improvement-based analyses to identify special-cause variation, adjusted analyses to generate standardized ratios and comparative plots for site comparisons. Analysis details are provided in Appendix 3 (available at www. cmajopen.ca/content/6/1/E44/suppl/DC1).

\section{Ethics approval}

The study was approved by the Mount Sinai Hospital Research Ethics Board. Individual sites will receive approval from local research ethics boards or quality-improvement committees, as appropriate.

\section{Interpretation}

Every year, more than 25000 infants are born prematurely in Canada, ${ }^{36}$ and most survive for more than 70 years. Thus, preterm birth affects the health care system more than any other chronic condition. Improving outcomes of preterm babies can improve quality of life substantially and reduce health care costs over the lifespan. The CPTBN will address existing gaps in maternal-perinatal-neonatal research and strive for outcome improvement by creating a transdisciplinary team working within an objectively structured performance-measurement framework.

\section{Conclusion}

The CPTBN will be a national network encompassing the spectrum of maternal-fetal-neonatal-childhood events that 
affect preterm infant outcomes. Our goals for the CPTBN are threefold: 1) to enable multidisciplinary evidence-based practice change that results in improved neonatal outcomes, 2) to evaluate and standardize preterm birth interventions by associating practices with improved outcomes and 3) to estimate the economic burden of preterm birth and the costeffectiveness of preterm birth interventions to improve the standard of care and guide efficient resource allocation.

\section{References}

1. Perinatal health indicators for Canada 2013: a report of the Canadian Perinatal Surveillance System. Ottawa; Public Health Agency of Canada; 2013.

2. Lim G, Tracey J, Boom N, et al. CIHI survey: hospital costs for preterm and small-for-gestational age babies in Canada. Healthc Q 2009;12:20-4.

3. Lee SK, Aziz K, Singhal N, et al. Improving the quality of care for infants: a cluster randomized controlled trial. CMA7 2009;181:469-76.

4. Lee SK, Shah PS, Singhal N, et al. Association of a quality improvement program with neonatal outcomes in extremely preterm infants: a prospective cohort study. CMA7 2014;186:E485-94.

5. Lee SK, Aziz K, Singhal N, et al. The Evidence-based Practice for Improving Quality method has greater impact on improvement of outcomes than dissemination of practice change guidelines and quality improvement training in neonatal intensive care units. Paediatr Child Health 2015;20:e1-9.

6. Shah PS, Seidlitz W, Chan P, et al. Internal audit of the Canadian Neonatal Network data collection system. Am 7 Perinatol 2017;34:1241-9.

7. Synnes A, Luu TM, Moddemann D, et al. Determinants of developmental outcomes in a very preterm Canadian cohort. Arch Dis Child Fetal Neonatal Ed 2017;102:F235-44.

8. Kenyon S, Pike K, Jones DR, et al. Childhood outcomes after prescription of antibiotics to pregnant women with spontaneous preterm labour: 7-year follow-up of the ORACLE II trial. Lancet 2008;372:1319-27.

9. O'Brien K, Bracht M, MacDonell K, et al. A pilot cohort analytic study of Family Integrated Care in a Canadian neonatal intensive care unit. BMC Pregnancy Childbirth 2013;13(Suppl 1):S12.

10. Lodha A, Seshia M, McMillan DD, et al. Association of early caffeine administration and neonatal outcomes in very preterm neonates. $7 A M A$ Pediatr 2015; 169:33-8.

11. Soraisham AS, Harabor A, Shivananda S, et al. Trends and variations in the use of inhaled nitric oxide in preterm infants in Canadian neonatal intensive care units. Am 7 Perinatol 2016;33:715-22.

12. Abstractor's manual. Version 3.0.0. Toronto: Canadian Neonatal Network; 2017. Available: www.canadianneonatalnetwork.org/Portal/LinkClick.aspx?fileticket= h4VXdWoqMNM\%3d\&tabid=69 (accessed 2017 Dec. 7).

13. Shah PS, Sankaran K, Aziz K, et al. Outcomes of preterm infants < 29 weeks' gestation over 10-year period in Canada: A cause for concern? 7 Perinatol 2012; 32:132-8.

14. Shah V, Warre R, Lee SK. Quality improvement initiatives in neonatal intensive care unit networks: achievements and challenges. Acad Pediatr 2013; 13(Suppl 6):S75-83.

15. Plsek PE. Quality improvement methods in clinical medicine. Pediatrics 1999; 103(Suppl E):203-14.

16. The Canadian Neonatal Network ${ }^{\mathrm{TM}}$ annual report 2014 rapport annuel. Toronto: Canadian Neonatal Network; 2014. Available: www.canadianneonatalnetwork. org/Portal/LinkClick.aspx?fileticket=eGgxmMubxjk\%3d\&tabid=39 (accessed 2018 Jan. 15).

17. Melamed N, Shah J, Soraisham A, et al. Association between antenatal corticosteroid administration-to-birth interval and outcomes of preterm neonates. Obstet Gynecol 2015;125:1377-84.

18. Melamed N, Shah J, Yoon EW, et al. The role of antenatal corticosteroids in twin pregnancies complicated by preterm birth. Am 7 Obstet Gynecol 2016;215: 482.e1-9.

19. Bustamante C, Valencia M, Torres C, et al. Effects of a single course of prenatal betamethasone on dendritic development in dentate gyrus granular neurons and on spatial memory in rat offspring. Neuropediatrics 2014;45:354-61.

20. Kuperman AA, Koren O. Antibiotic use during pregnancy: How bad is it? BMC Med 2016;14:91.

21. Tamburini S, Shen N, Wu HC, et al. The microbiome in early life: implications for health outcomes. Nat Med 2016;22:713-22.

22. Esplin MS. The importance of clinical phenotype in understanding and preventing spontaneous preterm birth. Am f Perinatol 2016;33:236-44.

23. Manuck TA, Esplin MS, Biggio J, et al. The phenotype of spontaneous preterm birth: application of a clinical phenotyping tool. Am 7 Obstet Gynecol 2015; 212:487.e1-11.

24. Wyckoff MH. Initial resuscitation and stabilization of the periviable neonate: the Golden-Hour approach. Semin Perinatol 2014;38:12-6.
25. Richardson DK, Corcoran JD, Escobar GJ, et al. SNAP-II and SNAPPE-II: simplified newborn illness severity and mortality risk scores. 7 Pediatr 2001; 138:92-100

26. Lee SK, Aziz K, Dunn M, et al. Transport Risk Index of Physiologic Stability, version II (TRIPS-II): a simple and practical neonatal illness severity score. $A m$ 7 Perinatol 2013;30:395-400.

27. AlFaleh K, Anabrees J. Probiotics for prevention of necrotizing enterocolitis in preterm infants. Evid Based Child Health 2014;9:584-671.

28. Rao SC, Athalye-Jape GK, Deshpande GC, et al. Probiotic supplementation and late-onset sepsis in preterm infants: a meta-analysis. Pediatrics 2016;137: e20153684.

29. Fischer HS, Bührer C. Avoiding endotracheal ventilation to prevent bronchopulmonary dysplasia: a meta-analysis. Pediatrics 2013;132:e1351-60.

30. Baraldi E, Filippone M. Chronic lung disease after premature birth. $N$ Engl 7 Med 2007;357:1946-55.

31. Jobe AH. The new BPD. NeoReviews 2006; 7:e531-45.

32. Schmölzer GM, Te Pas AB, Davis PG, et al. Reducing lung injury during neonatal resuscitation of preterm infants. 7 Pediatr 2008;153:741-5.

33. Johnston KM, Gooch K, Korol E, et al. The economic burden of prematurity in Canada. BMC Pediatr 2014;14:93.

34. Ananth CV, Friedman AM, Gyamfi-Bannerman C. Epidemiology of moderate preterm, late preterm and early term delivery. Clin Perinatol 2013;40:601-10.

35. Lachin JM. Biostatistical methods: the assessment of relative risks. New York: John Wiley \& Sons; 2000.

36. Table 102-4512 - Live births, by weeks of gestation and sex, Canada, provinces and territories, annual. Ottawa: Statistics Canada; 2017. Available: http://www5. statcan.gc.ca/cansim/a26? lang=eng\&id=1024512 (accessed 2018 Jan. 15).

Affiliations: Department of Paediatrics (Shah, O'Brien), Mount Sinai Hospital; Department of Pediatrics (Shah, O'Brien), University of Toronto, Toronto, Ont.; Departments of Obstetrics and Gynecology, Radiology, and Health Research Methods, Evidence, and Impact (McDonald), McMaster University, Hamilton, Ont.; Women and Babies Program (Barrett), Sunnybrook Health Sciences Centre, Sunnybrook Research Institute, Toronto, Ont.; Department of Pediatrics (Synnes, Shivananda), University of British Columbia, Vancouver, BC; Canadian Premature Babies Foundation (Robson, Foster), Toronto, Ont.; Women and Babies Program (Robson), Sunnybrook Health Sciences Centre, Toronto, Ont.; Department of Obstetrics and Gynecology (Pasquier), Université de Sherbrooke, Sherbrooke, Que.; Department of Obstetrics and Gynaecology (Joseph), University of British Columbia, Vancouver, BC; Department of Pediatrics (Piedboeuf), Université Laval, Québec, Que.; Department of Pediatrics (Lacaze-Masmonteil), Alberta Health Services and Cumming School of Medicine, University of Calgary, Calgary, Alta.; Department of Obstetrics and Gynecology (Chaillet), Université Laval, Québec, Que.; Child Health Evaluative Sciences (Pechlivanoglou), The Hospital for Sick Children, Toronto, Ont.

Contributors: All of the investigators conceived of the Canadian Preterm Birth Network concept. Prakesh Shah led the protocol design process and drafted the manuscript. Sarah McDonald, Jon Barrett, Anne Synnes, Kate Robson, Jonathan Foster, Jean-Charles Pasquier, K.S. Joseph, Bruno Piedboeuf, Thierry Lacaze-Masmonteil, Karel O'Brien, Sandesh Shivananda and Nils Chaillet participated in network and protocol design and will direct data collection. All of the authors gave final approval of the version to be published and agreed to be accountable for all aspects of the work.

Funding: The Canadian Preterm Birth Network is supported by Team Grant PBN150642 from the Canadian Institutes of Health Research (CIHR). K.S. Joseph and Prakesh Shah hold Applied Research Chairs in Reproductive and Child Health Services and Policy Research awarded by the CIHR (APR-126338 and APR-126340, respectively). Sarah McDonald is supported by a Tier II Canada Research Chair.

Acknowledgements: The authors thank all site investigators for their continuing support. They also thank Sarah Hutchinson and Natasha Musrap from the Maternal-Infant Care Research Centre, Mount Sinai Hospital, for editorial assistance in the preparation of the manuscript. Organizational support for the Canadian Preterm Birth Network was provided by the Maternal-Infant Care Research Centre.

Supplemental information: For reviewer comments and the original submission of this manuscript, please see www.cmajopen.ca/content/6/1/ E44/suppl/DC1. 